\title{
Biophysical Interface of Anti-Matter for Virtual Living in Real World: A Reality in Chemical Converted in Parallel Worlds
}

\author{
Majid Monajjemi 1,* ${ }^{1}$, Fatma Kandemirli ${ }^{2 \mathbb{D}}$, Hossein Sakhaeinia ${ }^{1}$, Fatemeh Mollaamin ${ }^{2} \mathbb{D}$ \\ Department of Chemical engineering, Central Tehran Branch, Islamic Azad University, Tehran, Iran \\ Department of Biomedical Engineering, Faculty of Engineering and Architecture, Kastamonu University, Kastamonu, \\ Turkey \\ * Correspondence: m_monajjemi@srbiau.ac.ir; maj.monajjemi@iauctb.ac.ir (M.M.);
}

Scopus Author ID 670181068

Received: 12.05.2021; Revised: 5.06.2021; Accepted: 8.06.2021; Published: 24.06.2021

\begin{abstract}
We are going to discuss biology and the biophysical phenomenon of the anti-matters, both experimentally and theoretically or philosophically. Anti-matter is used to treat cancer and thymine dimer problems due to UV radiation. Positrons are beamed through tissue at such velocity that they do not annihilate until they encounter cancer cells. Formed gamma rays damage the cancer tissue. Nowadays, anti-matter is used in positron emission tomography (PET). In evolutionary biology, where we see evidence that consciousness has emerged in at least humans, lifeforms can self-organize to exhibit collective emergent super-consciousness. In this work, we are looking at an ontological explanation of the relationship between the results obtained with the Ecology. The proton transfer mechanism is a critical phenomenon that is related to the acid-base characteristics of the nucleobases in Watson-Crick base pairs. They exhibited a strong probability for protons to change place within the hydrogen bond due to quantum tunneling, which will alter the genetic code and cause mutations. These mutations could be the cause of several diseases such as cancer. Probably, the quantum tunneling effect (such as proton transfer) appears in micro parallel worlds
\end{abstract}

Keywords: parallel worlds; anti-matter; quantum biology; proton tunnelling; DNA repairing.

(C) 2021 by the authors. This article is an open-access article distributed under the terms and conditions of the Creative Commons Attribution (CC BY) license (https://creativecommons.org/licenses/by/4.0/).

\section{Introduction}

In chemistry, the anti-matters have appeared as conjugate particles in the whole of mater. A few anti-maters are produced yearly at particle accelerators. The total productions are only less than one microgram/per year [1-7]. In cosmic ray collisions and some kinds of radioactivity phenomenon, a very small number of these have successfully been bounded together for forming anti-atoms. Basically, a particle and conjugate particles such as electron and positron have the same mass, but opposite charges and other differences can be mentioned in quantum eigenvalues $[1,2]$. The collision among these kinds of particles (mater and antimater) makes them annihilating and creating strong energy in the range of gamma rays. Occasionally, less-massive particle-antiparticle pairs of these energies appear only in ionizing radiation $\left(\mathrm{E}=\mathrm{mc}^{2}\right)$, and if the surrounding matter is present, the energy content of this radiation will be absorbed and converted into other forms of energy, such as heat or light $[3,4]$. Nevertheless, a super-stable or meta-stable of some kind position of antiparticles might exist, such as antiprotons in a normal matter, or it may be stored in superfluid helium [5-7]. 
Speculation is encouraged because helium is the only atom that theoretically cannot capture a low-energy antiproton [8]. It is known that anti-matter has the same kind of mass as anti-matter, but how do gravity influence matter and anti-matter? If we could have apples made of antimatter and drop them here on earth, would the apple go up? At the time, world-class scientists are trying to find funding for a project where they are trying to cool anti-matter molecules near absolute zero by lasers to research how gravity affects anti-matter. Anti-matter particles can be attached to each other and produce anti-matter, for instance, a positron ( $e+$ or" $\left.e^{\dagger "}\right)$ and an antiproton $\left(\mathrm{P}^{-}\right.$or" $\left.P^{\dagger "}\right)$ can be made an anti-hydrogen atom $\left(H^{\dagger}\right)$. Meanwhile, for all elements of the Mendeleev table, a conjugate element can be defined as an instance $\left(\mathrm{C}^{\dagger}, \mathrm{N}^{\dagger}, \mathrm{O}^{\dagger}, \mathrm{P}^{\dagger}\right.$, and $\left.\mathrm{Mg}^{\dagger}\right)$. Therefore there are conjugate molecules for each biomolecule, such as $G^{\dagger}$ as anti-guanine, $A^{\dagger}$ as anti-adenine, $C^{\dagger}$ as anti-cytosine and $T^{\dagger}$ as antithymine or even $D N A^{\dagger}$ as anti-DNA. Nevertheless, there are various speculations as to why the observable universe is seemingly almost completely matter, and this question might be answered scientifically, whether there exist other places that apparent anti-matter instead of universe materials. However, at this time, the apparent asymmetry of matter and anti-matter in the visible universe is one of the greatest unsolved problems in science and physical philosophy. The process by which this asymmetry between particles and antiparticles developed is called "Baryogenesis". Anti-Human can be defined as a human that made up of whole antiparticles, or in otherwise, to every fundamental particle in this kind of human there are correspond an antiparticle with opposite quantum eigenvalues (such as difference charge or spin and so on), but there are other identical properties (such as mass and chemical bonding). This work will discuss biology and the biophysical phenomenon of these kinds of anti-matters both experimentally and theoretically or philosophically. Anti-matter is used to treat cancer. Positrons are beamed through tissue in such velocity that they do not annihilate until they encounter cancer cells. Formed gamma rays damage the cancer tissue. A particle traveling through space can move in many different ways, curved, oscillating, and squiggly on time paths. Each of these paths has amplitude, and when summed up in a vector, all that remains are the comparably few histories that abide by the laws and forces of nature. Nowadays, anti-matter is used in positron emission tomography (PET). The PET device produces a three-dimensional picture of the functional processes in the body. It detects pair of gamma rays emitted by electron-positron annihilation.

\section{Materials and Methods}

\subsection{Theoretical background.}

2.1.1. The Dirac equation for anti-matter.

Particle energy can be defined as $E-V=\frac{\vec{P}^{2}}{2 m}$ (1), where $m$ and $\vec{P}$ are mass and momentum of the particles, respectively. Particles of low energies such as electrons and positrons in an atom can well be described by equation (1). Although particles with high energy (around $\mathrm{MeV}$ energies), same as electrons excited via accelerators, no longer obey from equation (1), a suitable equation by considering relativistic behavior can be interpreted the behavior of this kind of particles based on equation (2); $(E-V)^{2}=c^{2} \vec{P}^{2}+m_{0}^{2} c^{4} \&(E-$ $V)= \pm \sqrt{c^{2} \vec{P}^{2}+m_{0}^{2} c^{4}}\left(\right.$ 2). Conversion of (2) via considering the operators $\hat{E}=i \hbar \frac{\partial}{\partial t}$ and $\hat{P}^{2}=$ 
$-\hbar^{2} \nabla^{2}$, leads to $\left(i \hbar \frac{\partial}{\partial t}-V\right)^{2} \Psi=-\hbar^{2} c^{2} \nabla^{2} \Psi+m_{0}^{2} c^{4} \Psi$ (3). The consequence of equation (2) indicates that there are two types of energies, one is negative, and the other is positive, referring to the negative and positive kinetic segment of the total energy. Based on electron wave function as: $\Psi(x, t)=\operatorname{Sin}\left(P x-\frac{E}{\hbar} t\right)$ then the Dirac equation stated that: $\left(\frac{\partial}{\partial x}-\frac{1}{C} \frac{\partial}{\partial t}\right) \Psi_{+}=$ $\left(\frac{m C}{\hbar}\right) \Psi_{-}$and $\left(\frac{\partial}{\partial x}-\frac{1}{C} \frac{\partial}{\partial t}\right) \Psi_{-}=\left(\frac{m C}{\hbar}\right) \Psi_{+}$(4). The $\Psi_{+}$and $\Psi_{-}$, referring to the negative and positive kinetic segments of the total electron wave function. By a simple modification in equation (4), the Dirac equation can be written as: $\left(\frac{\partial}{\partial x^{2}}-\right.$ $\left.\frac{1}{C} \frac{\partial}{\partial t^{2}}\right) \Psi_{\mp}=\left(\frac{m C}{\hbar}\right) \Psi_{\mp}$ and by considering the relativistic, we have $\left(\vec{P}^{2}-\left(1 / c^{2}\right) E^{2}=m^{2} c^{2}\right.$.

The relativistic phenomenon indicates that electron energy can have positive and or negative, based on the Dirac equation, which means the energy of the electron (for positive amount) and the energy of positron or antielectron(for negative amount ). This is the first interpretation of anti-matter in nature. Materialism is an axiom that says matter and other physical things, such as light and space-time, are the fundamental substances of reality. In this view, the terms "abstract" and "physical" are juxtaposed, where abstract, i.e., not real, information merely describes the real physical things. The axiom does not explain where the physical stuff comes from. Dirac explained that all positive energies are greater than $\mathrm{mc}^{2}$ and all negative energies are less than $\mathrm{mc}^{2}$, and an energy gap between the two is $2 \mathrm{mc}^{2}$. Then, it seems possible that a negative energy electron could absorb a photon of energy greater than $2 \mathrm{mc}^{2}$ and make a jump to a positive energy state. A hole is then left in the previously occupied negative energy "sea" of electrons. At first, Dirac believed this hole to be a proton, but it was later shown that it would need to have all of the same properties of an electron but with a positive charge, which indicates positron. Due to the symmetrical and spherical universe, it is obvious that the Big Bang should have resulted in equal amounts of matter and anti-matter. On the other hand, as the total mutual annihilation did not take place ( as evidenced by our universe's existence), so the universe is now all matter, all original anti-matter made dark matter in the galaxy. In the interstellar or intergalactic, the particles and antiparticles start from being completely separated, residing in the vacuum of interstellar space. These are not devoid of competing particles because they have a much lower particle density than the original Big Bang. They do not suffer from being in a dense milieu of particles and antiparticles whose Coulomb attractions tend to cancel out their effects.

\subsubsection{Quantum biology perspective.}

Quantum biology is a subject of applied quantum mechanics and theoretical biophysical chemistry [9-11]. The main process in this field consists of chemical reactions, light absorption, formation of excited electronic states, transfer of excitation energy, and the transfer of electrons and protons (hydrogen ions), such as photosynthesis, olfaction, and cellular respiration. Quantum biology creates biological interactions in light of quantum mechanical effects via modeling and simulation. It also discusses the influence of non-trivial quantum phenomena, which can be interpreted by reducing the biological process towards a fundamental quantum theory based on speculative. Quantum biology is an emerging field nowadays that has been basically concentrated on those questions which are required any further conclusion than experimentation. Primary pioneers of quantum mechanics guessed about the important role of applications of quantum mechanics in the biological phenomenon. Erwin Schrödinger's book 
what is Life (1944), has been discussed quantum effects in biology. He further suggested that DNA mutations might be introduced via "quantum leaps". Other pioneers Niels Bohr, Pascual Jordan, and Max Delbruck, agreed that complementarities' quantum model was fundamental to the life sciences. In addition, Per-Olov Löwdin published a paper about proton tunneling as a major function for DNA mutation. He designed a fundamental idea as a novel research approach called "quantum biology"[10]. During cell reproduction, some when via the process of copying the DNA a mutation or an error in genetic codes can occur (Lowdin DNA mutation model )[10]. In this model, a nucleotide may change its form through a process of quantum tunneling [12-16].

Due to this change, some of the nucleotides will lose their abilities for pairing with each of the original opposite pairs and therefore appear to change the structure of the DNA strand. The UVA and also UVB and UVC radiations can modify the bonds along the DNA strand in the pyrimidine and cause them to bond with themselves, creating a dimer. In many prokaryotes, this kind of bond can be repaired to its original shape through a DNA repair enzyme photolysis[17-20]. In the mechanism of photolysis, light is needed for any further repairing of the strand that works with cofactor FADH. Photolysis can be excited via visible radiation and transfers an electron to the cofactor FADH. FADH gives this extra electron to the dimer bond for breaking and then repairing the DNA. This transfer is accomplished through the tunneling effect of the electron from the FADH to the dimer. Although the range of the tunneling effect is much larger than feasible in a vacuum, the tunneling in this phenomenon is called super exchange-mediated tunneling $[21,25]$.

\subsubsection{Root of physiology in space-time.}

Although the biological relativity concept is related to evolutionary development, the root of complex physiologic traits should appear within space-time phenomenon [26,27]. In the cellular-molecular phenomenon that biological behaviors give rise to physiologically traits, the evolution information from the present back to the protocell, then the spatial and temporal aspects of physiologic evolution can be analyzed according to the related boundaries [27,28]. For instance, the cholesterol (amazing molecule for the evolution of eukaryotes) was applied as an instrument to understand the evolution of cell-cell Communication, due to this fact that cholesterol requires eleven atoms of $\mathrm{O}_{2}$ to synthesize one molecule, confirming the requirement for atmospheric $\mathrm{O}_{2}$, which by starting of beginning in the Phanerozoic era, increasing and decreasing many times over the last few hundred million years [28,29]. By allowing for a thinning of the phospholipid bilayer, cholesterol causes the metabolism, mobility, and respiration in the cell membrane. Consequently, it facilitates cell-cell signaling via growth factors for giving rise to a formal endocrine system which is evidence for the causal relationship between cholesterol and eukaryotic evolution [29,30]. Cholesterol is the first initial of lung phospholipids (long \& short-terms in history) for the evolution of the gas exchanger from fish to amphibians, reptiles, birds, and mammals. Understanding epigenetic factors in these terms are by considering the quantum phenomena that the centrality of this quantum action is at the zygotic level via its unique means of biologic space-time.

Richard Feynman interpreted the quantum, known today as the "Sum over Histories" [31], explaining time as an orientation in space. This theory indicates that the probability of an event is confirmed via summing together all the possible histories of that event. A particle traveling through space can move in many different ways, curved, oscillating and squiggly on time paths. Each of these paths has amplitude, and when summed up in a vector, all that remains 
are the comparably few histories that abide by the laws and forces of nature. By other meaning, Sum over Histories indicates the direction of our ordinary clock time as simply a path in space that is more probable than any more exotic orientations time might have taken otherwise. Einstein, and Bohme similar Feynman, thought that time was an artifact that the past, present, and future all exist simultaneously. In Relativity [32], Einstein stated that "Since there exists in this four-dimensional structure (space-time) no longer any sections which represent 'now' objectively, the concepts of happening and becoming are indeed not completely suspended, but yet complicated. It seems, more image of physical reality as a four-dimensional existence, instead of, as hitherto, the evolution of a three-dimensional existence. Einstein proved that time is relative, not absolute, as classical Newtonian mechanics generally assumes. David Bohme [33] expresses two realities, 1- explicate and 2- implicate. Explication is the manner in which we conventionally perceive reality through the subjectivity of our evolved senses. Realities lie in another realm, implicate, which he describes as the singularly continuous fields. Here also, time is non-existent. Other worlds are just other directions in space within this system, some less probable, others equally as probable as the direction we experience. Here also, time is nonexistent. Within this system, other worlds are just other directions in space, some less probable, others equally as probable as the direction we experience. Stephen Hawking [34] said that "mostly the scientist makes a great mystery of the multi-universe (interpretation of the quantum theory of various worlds or parallel worlds), but to me, these are just different expressions of the Feynman path integral. Darwinism theory has no accommodation for this interpretation. Nevertheless, the zygotic uni-cell is explained as an epicenter of multicellular eukaryotic life that is completely capable of mitigating complex epigenetic inputs, and then a conceptual unification of quantum phenomena with biology is its output (Figure1).

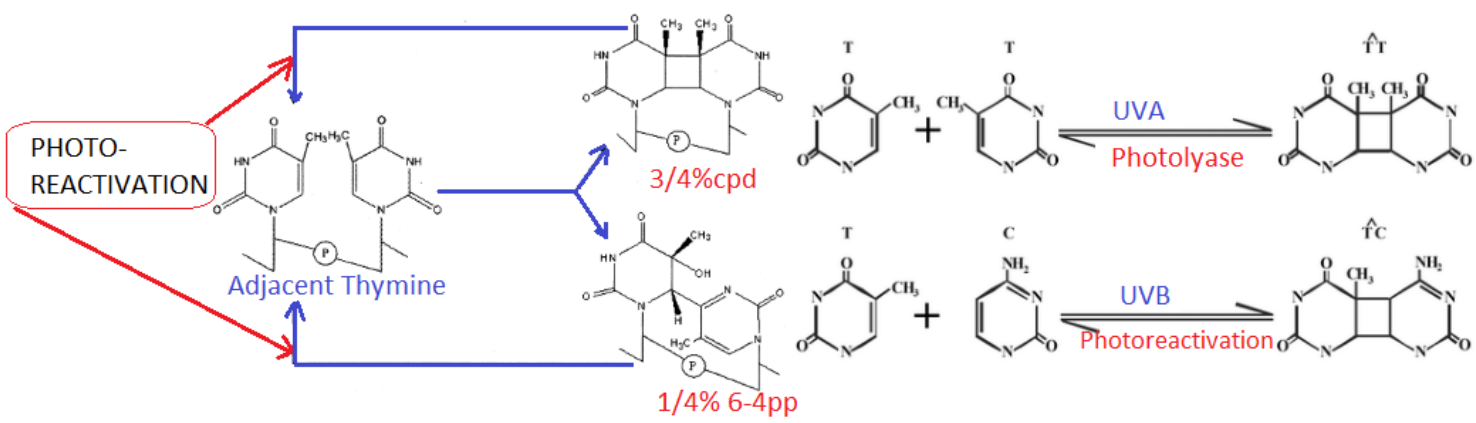

Figure 1. Repair of damaged DNA by photoreactivation mediated by the enzyme photolyase; UV radiation induces the formation of thymine dimmer on DNA having adjacent thymine bases.

\subsubsection{Splanchnic (Visceral) evolution.}

The biological system and initial molecules that have started lung evolution are structurally homologous with initial visceral tissue (root of phylogenetic and ontogenetic) [35]. For any more details, PTHrP signaling basically applies to the skin, kidney, skeleton and mediates homeostasis within the glomerulus and skeletal plasticity. In addition, by removing PTHrP, the function of lung alveoli, kidney, and skin barrier function are failed. It is notable that the PTHrP receptor gene duplicated during the water-land transition [36]. PTHrP signaling potential increased the bone plasticity based on Wolff's Law [37]. As discussed, lung surfactant has a mechanism that there are adequate surface activities to prevent the alveoli from collapsing on end-expiration when the lung expands. This process is regulated by the cell-cell signaling partners, PTHrP and leptin [37]. 


\subsubsection{Quantum tunneling in biology.}

An enzyme is described as a workhorse of any cell. Those are responsible for biosynthetic processes in the whole of dynamics phenomenon in biosystems, such as protein folding, gene repairing, and DNA replication. For all reactions catalyzed through enzymes, some of the main important factors can be categorized in hydrogen transfer (H-transfer) reactions, including proton, $\mathrm{H}$-atom, and hydride transfers. The importance of nuclear quantum tunneling in these reactions has been hotly debated for several decades [38], but it is well established that H-transfer reactions - whether enzymatic or unanalyzed - involve several degrees of H-tunneling due to the small mass of the proton.

\subsubsection{Proton tunneling \& spontaneous mutagenesis.}

There are several environmental items that can compromise the integrity of the genome and induce mutations during replication, including UV light, thymine dimer, ionizing radiation, and reactive oxygen species [39]. They hypothesized that since each of the four bases can appear in two readily interconvertible tautomer forms, A.C and G.T mismatches could occur if one of the bases adopts its rare and energetically less favorable form. In addition, the geometry of the canonical A-T and G-C WC base pairs is almost identical to the geometry of those tautomer WC-like mismatches, which could thus be accommodated in the binding pocket of the active site of a DNA polymerase (Figure 2).

(a)

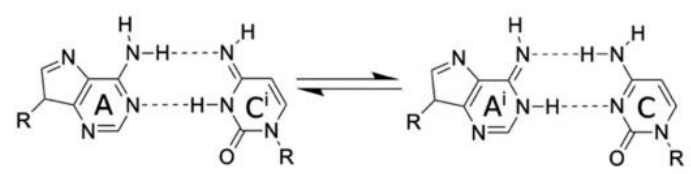

(b)
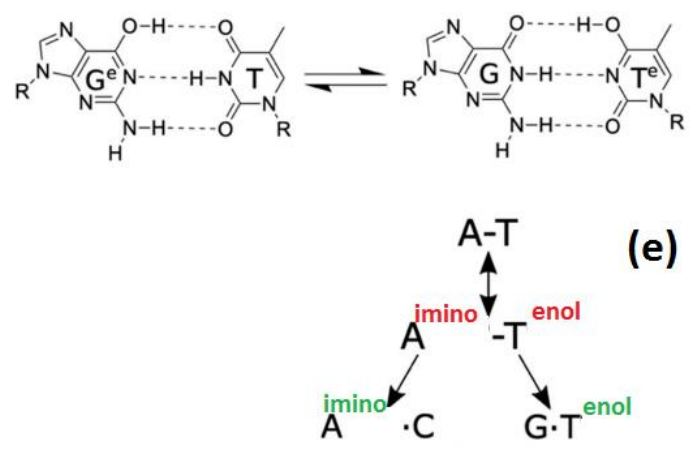

(e) (c)

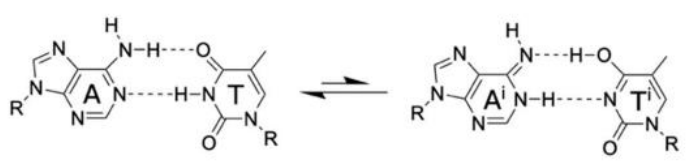

(d)

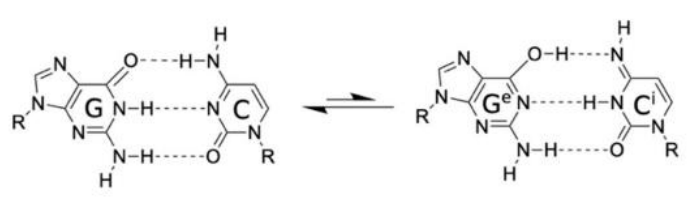

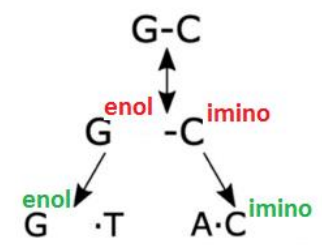

$\mathrm{H}$

Figure 2. (a,b), Watson-Crick (WC) hypothesis of spontaneous mutagenesis: WC-like A.C and G·T mismatches that preserve the geometry of canonical WC base pairs and can lead to spontaneous point mutations. (c-e), Löwdin's hypothesis of spontaneous mutagenesis.

The energies of the amino-keto and imino-enol forms of the A-T and G-C base pairs have been extensively modeled with ab-inito of DFT and correlation methods. The resulting double well configuration, representing the canonical and tautomer state, allows the proton to be transferred via quantum mechanical tunneling or classical over-the-barrier transfer. In 1965, Förster exhibited three energy transfers among donor and acceptor fluorophores that share common atomic transitions: "strong", intermediate "weak" and "very weak" coupling [40]. The energy transfers are defined as the reciprocal time required from an excited-state donor to a ground-state acceptor. During the interaction, the Columbic coupling between absorption and emission dipoles is based on the distance around $r^{-3}$ between donor and acceptor situation. The strong coupling strength results in major alterations of the absorption spectrum and is thought 
to be possible to mediate excitation energy transfer up to $100 \mathrm{~nm}$. In the weak intermediate coupling, transfer energies are comparable to that of vibrational relaxation. The coupling strength between fluorophores within this regime is expected to lead to minor alterations in the absorption spectrum, such as the Davydov splitting of certain vibronic bands. In the very weak coupling regime, the coupling strength is much lower than the vibronic bandwidth so that vibrational relaxation takes place before energy transfer. In this regime, fluorophores act independently so that it is expected that there is no alteration of the absorption spectrum. In addition, energy transfer is thought to be incoherent via FRET and is typically limited by a distance up to $10 \mathrm{~nm}$. The interaction is inversely proportional to the six power of the distance between donor and acceptor $r^{-6}$. In 1974, Knox and Kenkre formalized the slow de-phasing coherent energy transfer theory based on the unification of the strong and weak coupling theories [41].

\subsubsection{Simulation methodology.}

The thymine-thymine dimers interactions are explained via Lennard-Jones potentials as follows: $V_{L J}\left(r_{i j}\right)=4 \varepsilon_{i j}\left\{\left[\left(\frac{\sigma_{i j}}{r_{i j}}\right)^{12}-\left(\frac{\sigma_{i j}}{r_{i j}}\right)^{6}\right]\right\}, r<R_{C}$ (1). The Lorentz-Berthelot rule has been used for the inter forces among atoms as follows " $\sigma_{i j}=0.5\left(\sigma_{i}+\sigma_{j}\right)$ "and $\varepsilon_{i j}=\sqrt{\varepsilon_{i}}$ * $\sqrt{\varepsilon_{j}}$. (2). the non-bonded and bonded data, including van der Waals of related force fields, are listed in Tables1\&2. The total energies of the model systems are a total of several partial energies as follows: $E_{\text {(system) }}=\mathrm{E}_{\text {(bond) }}+\mathrm{E}_{\text {(angle) }}+\mathrm{E}_{\text {(torsion) }}+\mathrm{E}_{(\text {over })}+\mathrm{E}_{(\mathrm{vdW})}+\mathrm{E}_{(\text {(oulomb) }}+\mathrm{E}_{\text {(Specific) }}$, (3), where $\mathrm{E}_{\text {(bond) }}$ and ( $\left.\mathrm{E}_{\text {(angle) }}+\mathrm{E}_{\text {(torsion) })}\right)$ are bond formation and angle (both strain and torsional) energies, respectively. $\mathrm{E}_{(\text {over) }}$ is associated with valence and torsional angles, respectively, that prevent the over-coordination of the atoms. $\mathrm{E}_{\mathrm{vdW}}+\mathrm{E}_{(\mathrm{Coulomb})}$ are the dispersive and electrostatic energies contribution between all atoms, respectively. Finally, $\mathrm{E}_{\text {(Specific) }}$ is system-specific energy such as lone-pair, conjugation and hydrogen bonding.

Table1. Non-bonded parameters in terms of $\mathrm{E}_{\text {(van der waals) }}+\mathrm{E}_{\text {(Coulomb) }}$ Non bonded interaction

\begin{tabular}{l|l|c|c}
\hline \multicolumn{2}{c}{$V_{L J}\left(r_{i j}\right)=4 \varepsilon_{i j}\left\{\left[\left(\frac{\sigma_{i j}}{r_{i j}}\right)^{12}-\left(\frac{\sigma_{i j}}{r_{i j}}\right)^{6}\right]\right\}, r<R_{C}$} \\
\hline Atom type & Mass(g/mol) & $\boldsymbol{\sigma}(\boldsymbol{n m})$ & $\boldsymbol{\varepsilon ( \frac { \boldsymbol { k c a l } } { \boldsymbol { m o l } } )}$ \\
\hline $\mathrm{CH} 2$ & 14.03 & 0.396 & 0.0 .091 \\
\hline $\mathrm{C}=\mathrm{O}$ & 28.05 & 0.435 & 0.109 \\
\hline $\mathrm{O}-\mathrm{H}$ & 17.08 & 0.223 & 0.024 \\
\hline $\mathrm{C}-\mathrm{O}$ & 28.07 & 0.412 & 0.102 \\
\hline $\mathrm{C}-\mathrm{C}$ & 24.03 & 0.123 & 0.072 \\
\hline $\mathrm{N}-\mathrm{C}$ & 26.01 & 0.446 & 0.055
\end{tabular}

Table2. Parameters of bonded interactions of the atomistic force field. Bonded \& angle interaction

\begin{tabular}{|c|c|c|c|c|c|c|c|c|c|}
\hline $\multicolumn{10}{|c|}{\left[V_{b}\left(r_{i j}\right)=\sum_{\text {bonds }} k_{i j}^{b}\left(r_{i j}-b_{i j}\right)^{2}\right]}+\left\{\left[V_{\beta}\left(\theta_{i j k}\right)=0.5 \sum_{\text {angle }} k_{i j k}^{\theta}\left(\theta_{i j k}-\theta_{i j k}^{0}\right)^{2}\right]\right\}+\left\{\left[V\left(\varphi_{i j k l}\right) k_{\varphi}(1+\operatorname{Cos}(n \varphi-\delta)\right.\right.$ \\
\hline bond & $\mathbf{b}(\AA)$ & $\begin{array}{c}k^{b} \\
\mathrm{kcal} / \mathrm{mol}^{*} \AA^{2}\end{array}$ & angle & $\theta_{i j k}^{0}$ & $\begin{array}{c}\boldsymbol{k}_{i j k}^{\theta} \\
\left(\frac{\mathrm{kcal}}{\operatorname{mol}} * \operatorname{Rad}^{2}\right)\end{array}$ & $\begin{array}{r}\text { Dihedral } \\
\varphi_{i j k l}\end{array}$ & $\begin{array}{c}k_{\varphi} \\
\mathrm{kcal} / \mathrm{mol}\end{array}$ & $n$ & $\bar{\delta}$ \\
\hline $\mathrm{C}-\mathrm{H}$ & 1.11 & 330 & C-O-C & 117.2 & 53.1 & C-C-C-O & 0.32 & 1 & 0.00 \\
\hline $\mathrm{C}=\mathrm{O}$ & 1.21 & 340 & C-C-C & 122.1 & 65.9 & H-C-C-C & 0.400 & 3 & $\begin{array}{l}180 . \\
0\end{array}$ \\
\hline $\mathrm{O}-\mathrm{H}$ & 1.09 & 360 & $\mathrm{O}-\mathrm{C}-\mathrm{O}$ & 119.4 & 45.3 & O-C-C-H & 0.25 & 2 & 0.00 \\
\hline $\mathrm{C}-\mathrm{O}$ & 1.41 & 230 & C-C-H & 110.1 & 46.5 & C-O-C-H & 0.54 & 2 & 0.00 \\
\hline
\end{tabular}




\begin{tabular}{|c|c|c|c|c|c|c|c|c|c|}
\hline \multicolumn{10}{|c|}{ Bonded \& angle interaction } \\
\hline $\multicolumn{10}{|c|}{\left[V_{b}\left(r_{i j}\right)=\sum_{\text {bonds }} k_{i j}^{b}\left(r_{i j}-b_{i j}\right)^{2}\right]}+\left\{\left[V_{\beta}\left(\theta_{i j k}\right)=0.5 \sum_{\text {angle }} k_{i j k}^{\theta}\left(\theta_{i j k}-\theta_{i j k}^{0}\right)^{2}\right]\right\}+\left\{\left[V\left(\varphi_{i j k l}\right) k_{\varphi}(1+\operatorname{Cos}(n \varphi-\delta)\right.\right.$ \\
\hline bond & $\mathbf{b}(\AA)$ & $\begin{array}{c}k^{b} \\
\mathrm{kcal} / \mathrm{mol}^{*} \AA^{2}\end{array}$ & angle & $\theta_{i j k}^{0}$ & $\begin{array}{c}\boldsymbol{k}_{i j k}^{\theta} \\
\left(\frac{\mathbf{k c a l}}{m o l} * \operatorname{Rad}^{2}\right)\end{array}$ & $\begin{array}{r}\text { Dihedral } \\
\varphi_{i j k l}\end{array}$ & $\begin{array}{c}k_{\varphi} \\
\mathrm{kcal} / \mathrm{mol}\end{array}$ & $n$ & $\delta$ \\
\hline $\mathrm{C}-\mathrm{C}$ & 1.52 & 440 & H-C-H & 107.2 & 65.4 & H-C-C-H & 0.32 & 1 & $\begin{array}{l}180 . \\
0\end{array}$ \\
\hline $\mathrm{C}=\mathrm{C}$ & 1.34 & 365 & $\mathrm{O}-\mathrm{C}-\mathrm{H}$ & 108.2 & 44.7 & $\mathrm{O}-\mathrm{C}-\mathrm{C}-\mathrm{O}$ & 0.64 & 3 & 0.00 \\
\hline
\end{tabular}

The OPLS Lennard-Jones parameters for nucleic acid bases are included in Table 3.

Table3. OPLS Lennard-Jones parameters for nucleic acid bases.

\begin{tabular}{l|l|l} 
Atom & \multicolumn{1}{|c|}{$\boldsymbol{\sigma}, \AA$} & \multicolumn{1}{c}{$\boldsymbol{\varepsilon}, \boldsymbol{k c a l} / \mathbf{m o l}$} \\
\hline $\mathrm{O}$ & 2.95 & 0.205 \\
\hline $\mathrm{N}$ & 3,21 & 0.165 \\
\hline $\mathrm{C}$ in $\mathrm{C}=\mathrm{O}$ & 3.8 & 0.110 \\
\hline $\mathrm{C}$ normal & 3.5 & 0.085 \\
\hline $\mathrm{H}(\mathrm{N})$ & 0.0 & 0.0 \\
\hline $\mathrm{H}(\mathrm{C})$ & 2.25 & 0.07 \\
\hline $\mathrm{H}(\mathrm{O})$ & 1.95 & 0.05
\end{tabular}

All modeling and simulation details have been done based on the simulation program of CHARMM and our previous works [42-59].

\section{Results and Discussion}

Gene mutation. Genotyping of the exon 35 mutation has been simulated for clearing the mechanism of mutation in Cyt $c$, Fig.3. Mootha et al. exhibited a mutation that was performed by primer extension of PCR and then detected by matrix-assisted laser desorption ionization. PCR 5-AGCGGATAACTCAGAAACCT TCACTTACTG-3 and 5AGCGGATAACGGAACA ACAACAAAATCGGG-3 and homogeneous Mass Extend (5AATAATGTTTTAATTTTTAGAGAT3 primers were designed by using Spect- Designer. PCR products were purified using the shrimp alkaline phosphatase method and extended by the addition of a homogeneous Mass-Extend primer as per the Sequencer- Mass-Array protocol. We investigated our model with a wide range of megabase regions of that genome containing several definite genes and some other unknown genes through translation and expression data of m- RNA. The results are confirmed with experimental data. As improvement genes with higher-density m-RNA expression and more comprehensive protein-membrane interaction become available, it should be able to apply such analysis widely.

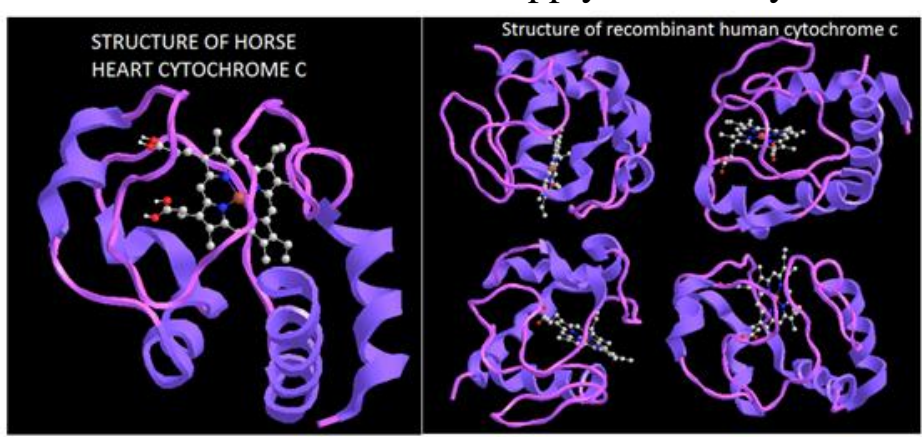

Figure 3. Optimized structure of horse heart and recombinant human cytochrome C.

The complex structures of 1rys, and 1skw (Figure 4) are predicted through dockings modeling and calculated via docking and Charmm software. The interaction energy analysis indicated the hydrophobic and hydrophilic contributions of related nucleic acids to the Gibbs free energies required for the complex formations. Some of the charged residues exhibited a 
large unfavorable depending on non-solvation interactions that were canceled out by large favorable columbic interaction, resulting in the destabilization of the structures. The free energies of destabilizations are compensated by the van der Waals interactions contemplated by hydrophobic amino acids for giving the stabilizing complexes. Since DNA absorbs wavelengths in the lower part of the UVB band, UV increases the thymine dimer band stronger. Overall, thymine dimer formation in the diatom of human skin on DNA is mainly induced by wavelengths below $320 \mathrm{~nm}$. The free energy differences between two states 1 and 2 of a system can be derived from classical statistical mechanics allowing us to express this function as:

$$
A_{2}-A_{1}=-R T \ln <\exp \left[-\frac{E_{2}-E_{1}}{R T}\right]>
$$

$\left(E_{2}-E_{1}\right)$ is the potential energy differences. The computed free energies are presented in Table 4.

Table 4. Solvation-free energies.

\begin{tabular}{l|l|l|l|l} 
Method & Adenine & Guanine & Cytosine & Thymine \\
\hline OPLS,TIP3 & -38.44 & -45.88 & -40.24 & -41.2 \\
\hline Charmm & -33.23 & -33.47 & -31.2 & -33.3 \\
\hline OPLF/FEP & -11.8 & -22.1 & -23.1 & -14.1 \\
\hline Amber/FEP & -12.6 & -18.9 & -13.1 & -9.33 \\
\hline Amber/TI & -12.4 & -22.1 & -17.99 & -11.89 \\
\hline AM1/SM2 & -19.33 & -24.5 & -18.55 & -13.22 \\
\hline QM/MM & -5.9 & -12.9 & -16.33 & -8.44 \\
\hline SCRF(6-31g*) & -6.11 & -16.3 & -13.44 & -8.45 \\
\hline FDPB & -10.3 & -18.8 & -16.45 & -10.32
\end{tabular}

The DFT and HF calculations of the thymine dimer exhibited that the ring fusion at the C5 and C6 atoms of two thymine bases produced a four-member cyclo-butane puckered ring. As well as a feature is seen with the $\mathrm{MP}_{\mathrm{n}}$ or Moller-Pleset level. This puckering leads to axial or equatorial positions for the replaced atoms on this ring and the atoms on the two thymine bases differ in their orientations (Particular in the orientation of the H6A atom. This 4-member is puckered, as in the neutral forms. The relative orientations of the H6A atom in TTp-1 are the same as the neutral $\mathrm{T}<>\mathrm{T}$.
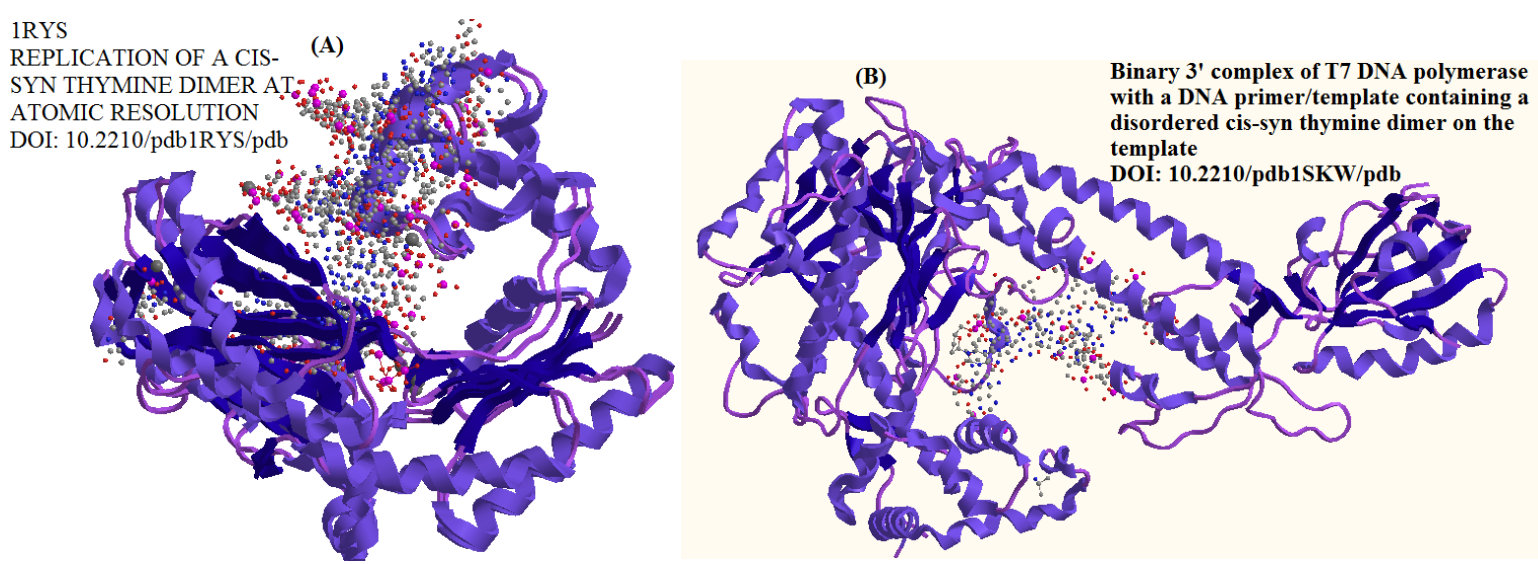

Figure 3. (A) Replication of a cis-syn thymine dimer; (B) Binary 3' complex of T7 DNA polymerase with a DNA primer/template containing a disordered cis-syn thymine dimer.

Electrons on the C6A-H6A bond participate in the stabilization of the $\pi$ system. The puckering of the cyclo-butane ring is responsible for the difference in the electronic configuration in the two thymine rings and makes a major order in producing the dissociation path in the thymine dimer. The UV radiations between $360 \mathrm{~nm}$ to $200 \mathrm{~nm}$ have been 
investigated for the study of thymine dimers. The small-term in the UVA band $(360 \mathrm{~nm})$ corresponds with the action spectrum for skin cancer induction. Growth reductions of human cells were also most pronounced in the UVB region. Therefore similarity between wavelengthdependent growth rate reduction and thymine dimer formation was high, especially in the region below $310 \mathrm{~nm}$. The overall similarity suggests that growth inhibition can be explained to thymine dimer formation combined with an insufficient repair. Since photo repair of thymine dimers is induced by wavelengths between 310 and $400 \mathrm{~nm}$, the ratio between UVB, UVA, and PAR determines whether damage and repair are in balance. In the light of this notion, growth rate reduction in the UVB region was caused by the unbalance between thymine dimer formation and repair [60-82].

\section{Conclusions}

quantum biology is a growing subject of interdisciplinary science, including quantum, biology, biochemistry, mathematics, engineering, and nanobiotechnology. Although it is a certain approach that the molecular machinery of life involves quantum phenomena such as coherence, tunneling, or entanglement, establishing a biological function for these quantum features remains a challenge. This challenge of the future will be to transfer measurable quantum biological systems into the more tractable workhorses of molecular biology and synthetic biologies, such as E. coli or yeast, to engineer systems that allow simultaneous detection of quantum-level events and their macroscopic outcomes. The Docking methods have allowed researchers to draw out a suitable complex between real biology and virtual simulation, such as thymine dimer, whose transient character makes it difficult for an experimental investigation. The regulation of proton tunneling has opened a new area of research in bioelectronics and biocomputing as well. This effect is applied as a "control gate in biocomputing" and realized by many enzymatic reactions. As the tunneling in semiconductors is sensitive to their environment, the devices can be used as sensors. The enzyme tunneling of protons is sensitive to the temperature, external pressure, and others, so the focus should be toward the studies of external parameters such as the magnetic field, electromagnetic wave frequency and intensity, etc., which will open new prospects in control and information transfer in bioelectronics.

\section{Funding}

This research received no external funding.

\section{Conflicts of Interest}

The authors declare no conflict of interest.

\section{References}

1. Agakishiev, H.; Observation of the anti-matter helium-4 nucleus. Nature 2011, 473, 353-356, https://doi.org/10.1038/nature10079.

2. Smorra, C.; A parts-per-billion measurement of the antiproton magnetic moment. Nature 2017, 550, 371374, https://doi.org/10.1038/nature24048.

3. Sala, S.; Ariga, A.; Ereditato, A.; Ferragut, R.; Giammarchi, M.; Leone, M.; Pistillo, C.; Scampoli, P. First demonstration of anti-matter wave interferometry". Science Advances 2019, 5, 7610, https://doi.org/10.1126/sciadv.aav7610. 
4. Schirber, M. Synopsis. More Dark Matter Hints from Cosmic Rays? . Physical Review Letters 2014, 113, 121102, https://doi.org/10.1103/PhysRevLett.113.121102.

5. Joshua Sokol, Giant space magnet may have trapped antihelium, raising idea of lingering pools of anti-matter in the cosmos . Science 2017, https://doi.org/10.1126/science.aal1067.

6. Sellner, S.; Besirli, M.; Bohman, M.; Borchert, M. J.; Harrington, J.; Higuchi, T.; Mooser, A.; Nagahama, H.; Schneider, G.; Smorra, C.; Tanaka, T.; Blaum, K.; Matsuda, Y.; Ospelkaus, C.; Quint, W.; Walz, J.; Yamazaki, Y.; Ulmer, S. Improved limit on the directly measured antiproton lifetime, New Journal of Physics 2017, 19, 083023, https://doi.org/10.1088/1367-2630/aa7e73.

7. Gibney, E. Physicists plan anti-matter's first outing - in a van". Nature 2018, 554, 412-413, https://doi.org/10.1038/d41586-018-02221-9.

8. Gsponer, A.; Hurni,J.P. Antimatter induced fusion and thermonuclear explosions. Independent Scientific Research Institute 2006, 1-10, https://arxiv.org/abs/physics/0507125.

9. Hing, G.B.; Siang,T.E.; Priyia, P. Quantum Biology: Does quantum physics hold the key to revolutionizing medicine, Progress in Drug Discovery \& Biomedical. Science 2020,3, https://doi.org/10.36877/pddbs.a0000130.

10. Frank, T.; Quantum Tunnelling to the Origin and Evolution of Life. Current Organic Chemistry 2013, 17, 1758-1770, https://doi.org/10.2174/138527281131799900083.

11. Cao, J. ; Cogdell, R.J.; Coker, D.F.; Duan, H.G.; Hauer, J.; Kleinekathöfer, U.; Jansen, T. L.C.; Mančal, T.; Miller, R.J. D.; Ogilvie, J. P.; Prokhorenko, V. I.; Renger, T.; Tan, H.S.; Tempelaar, R.; Thorwart, M.; Thyrhaug, E.; Westenhoff, S.; Zigmantas, D. "Quantum Biology Revisited". Science Advances 2020, 6, eaaz4888, https://doi.org/10.1126/sciadv.aaz4888.

12. Offenbacher, A.R.; Sharma, A.; Doan, P.E.; Klinman, J.P.; Hoffman, B.M. The Soybean LipoxygenaseSubstrate Complex: Correlation between the Properties of Tunneling-Ready States and ENDOR-Detected Structures of Ground States. Biochemistry 2020, 59, 901-910, https://doi.org/10.1021/acs.biochem.9b00861.

13. Dong, C.S.; Zhang, W.L.; Wang, Q.; Li, Y.S.; Wang, X.; Zhang, M.; Liu, L. Crystal structures of cyanobacterial light-dependent protochlorophyllide oxidoreductase. Proc. Natl. Acad. Sci. USA 2020, 117, 8455-8461, https://doi.org/10.1073/pnas.1920244117.

14. Tao, M.-J.; Zhang, N.-N.; Wen, P.-Y.; Deng, F.-G.; Ai, Q.; Long, G.-L. Coherent and incoherent theories for photosynthetic energy transfer. Sci. Bull. 2020, 65, 318-328, https://doi.org/10.1016/j.scib.2019.12.009.

15. Cao, J.; Cogdell, R.J.; Coker, D.F.; Duan, H.-G.; Hauer, J.; Kleinekathöfer, U.; Jansen, T.L.; Man`cal, T.; Miller, R.D.; Ogilvie, J.P. Quantum biology revisited. Sci. Adv. 2020, 6, eaaz4888, https://doi.org/10.1126/sciadv.aaz4888.

16. Proppe, A.H.; Li, Y.C.; Aspuru-Guzik, A.; Berlinguette, C.P.; Chang, C.J.; Cogdell, R.; Doyle, A.G.; Flick, J.; Gabor, N.M.; van Grondelle, R. Bioinspiration in light harvesting and catalysis. Nat. Rev. Mater. 2020, 5, 828-846, https://doi.org/10.1038/s41578-020-0222-0.

17. Freixas, V.; Tretiak, S.; Makhov, D.V.; Shalashilin, D.V.; Fernandez-Alberti, S. Vibronic Quantum Beating between Electronic Excited States in a Heterodimer. J. Phys. Chem. B 2020, 124, 3992-4001, https://doi.org/10.1021/acs.jpcb.0c01685.

18. Lambrev, P.H.; Akhtar, P.; Tan, H.-S. Insights into the mechanisms and dynamics of energy transfer in plant light-harvesting complexes from two-dimensional electronic spectroscopy. Biochim. Biophys. Acta Bioenerg. 2020, 1861, 148050, https://doi.org/10.1016/j.bbabio.2019.07.005.

19. Lukzen, N.N.; Ivanov, K.L.; Sadovsky, V.M.; Sagdeev, R.Z. Magnetic field effect on recombination of radicals diffusing on a two-dimensional plane. J. Chem. Phys. 2020, 152, 034103, https://doi.org/10.1063/1.5131583.

20. Babcock, N.S.; Kattnig, D.R. Electron-Electron Dipolar Interaction Poses a Challenge to the Radical Pair Mechanism of Magnetoreception. J. Phys. Chem. Lett. 2020, 11, 2414-2421, https://doi.org/10.1021/acs.jpclett.0c00370.

21. Patel, A.D. Grover's Algorithm in Natural Settings. arXiv 2020, arXiv:2001.00214, https://arxiv.org/abs/2001.00214.

22. Tolosa, S.; Sansón, J.; Hidalgo, A. Mechanisms of the TA to CG transition studied by SMD simulations: Deamination vs. tautomerisation. J. Mol. Liq. 2020, 308, 113036, https://doi.org/10.1016/j.molliq.2020.113036. 
23. Li, P.; Rangadurai, A.; Al-Hashimi, H.M.; Hammes-Schiffer, S. Environmental Effects on Guanine-Thymine Mispair Tautomerization Explored with Quantum Mechanical/Molecular Mechanical Free Energy Simulations. J. Am. Chem. Soc. 2020, 142, 11183-11191, https://doi.org/10.1021/jacs.0c03774.

24. Qaswal, A.B. Quantum Electrochemical Equilibrium: Quantum Version of the Goldman-Hodgkin-Katz Equation. Quantum Rep. 2020, 2, 17, https://doi.org/10.3390/quantum2020017.

25. Qaswal, A.B. Lithium stabilizes the mood of bipolar patients by depolarizing the neuronal membrane via quantum tunneling through the sodium channels. Clin. Psychopharmacol. Neurosci. 2020, 18, 214, https://doi.org/10.9758/cpn.2020.18.2.214.

26. Torday, J.; Rehan, V. Evolutionary Biology, Cell-Cell Communication and Complex Disease. Wiley: Hoboken, NJ, USA 2012, https://doi.org/10.1002/9781118130452.

27. Orgeig, S.; Daniels, C.; Johnston, S.; Sullivan, L. The pattern of surfactant cholesterol during vertebrate evolution and development: Does ontogeny recapitulate phylogeny? Reprod. Fertil. Dev. 2003, 15, 55-73, https://doi.org/10.1071/rd02087.

28. Daniels, C.; Orgeig, S.; Sullivan, L.; Ling, N.; Bennett, M.; Schürch, S.; Val, A.; Brauner, C. The origin and evolution of the surfactant system in fish: Insights into the evolution of lungs and swim bladders. Physiol. Biochem. Zool. 2004, 77, 732-749, https://doi.org/10.1086/422058.

29. Maina, J. Fundamental structural aspects and features in the bioengineering of the gas exchangers: Comparative perspectives. Adv. Anat. Embryol. Cell Biol. 2002, 163, 1-108, https://doi.org/10.1007/978-3642-55917-4.

30. West, J. Thoughts on the pulmonary blood-gas barrier. Am. J. Physiol. Lung Cell Mol. Physiol. 2003, 285, L501-L513, https://doi.org/10.1152/ajplung.00117.2003.

31. Feynman, R.; Hibbs, A.; Styer, D. Quantum Mechanics and Path Integrals; Dover Publications: Mineola, NY, USA 2010, https://www.amazon.com/Quantum-Mechanics-Path-Integrals-Emended/dp/0486477223.

32. Einstein, A. The Principle of Relativity; Dover Publications: New York, NY, USA 1952, https://www.amazon.com/Principle-Relativity-Dover-Books-Physics/dp/0486600815.

33. Bohm, D. Wholeness and the Implicate Order; Routledge and Kegan Paul: New York, NY, USA 1980, https://www.amazon.com/Wholeness-Implicate-Order-David-Bohm/dp/0415289793.

34. Hawking, S. Cosmology from the top down. In Universe or Multiverse; Cambridge University Press: Cambridge, UK, USA 2009.

35. Torday, J.; Rehan, V. Lung evolution as a cipher for physiology. Physiol. Genom. 2009, 38, 1-6, https://doi.org/10.1152/physiolgenomics.90411.2008.

36. Pinheiro, P.; Cardoso, J.; Power, D.; Canário, A. Functional characterization and evolution of PTH/PTHrP receptors: Insights from the chicken. BMC Ecology and evolution 2012, 12, https://doi.org/10.1186/14712148-12-110.

37. Ruff, C.; Holt, B.; Trinkaus, E. Who's afraid of the big bad Wolff?: "Wolff's law" and bone functional adaptation. Am. J. Phys. Anthropol. 2006, 129, 484-498, https://doi.org/10.1002/ajpa.20371.

38. Johannissen, L.O.; Iorgu, A.I.; Scrutton, N.S.; Hay, S. What are the signatures of tunnelling in enzymecatalysed reactions? Faraday Discuss. 2019, 221, 367-378, https://doi.org/10.1039/C9FD00044E.

39. Chatterjee, N.; Walker, G.C. Mechanisms of DNA damage, repair, and mutagenesis. Environ. Mol. Mutagenes. 2017, 58, 235-263, https://doi.org/10.1002/em.22087.

40. Förster, T. Delocalized Excitation and Excitation Transfer; Sinanoglu, O., Ed.; Modern Quantum Chemistry. Istanbul Lectures 3; Academic Press: New York, NY, USA; London, UK 1965, https://www.osti.gov/servlets/purl/4626886.

41. Kenkre, V.; Knox, R. Theory of fast and slow excitation transfer rates. Phys. Rev. Lett. 1974, 33, 803, https://doi.org/10.1103/PhysRevLett.33.803.

42. Monajjemi, M. Cell membrane causes the lipid bilayers to behave as variable capacitors: A resonance with self-induction of helical proteins. Biophysical Chemistry 2015, 207, 114-127, https://doi.org/10.1016/j.bpc.2015.10.003.

43. Monajjemi, M. Liquid-phase exfoliation (LPE) of graphite towards graphene: An ab initio study. Journal of Molecular Liquids 2017, 230, 461-472, https://doi.org/10.1016/j.molliq.2017.01.044.

44. Monajjemi, M.; Naderi, F.; Mollaamin, F.; Khaleghian, M. Drug design outlook by calculation of second virial coefficient as a nano study. Journal of the Mexican Chemical Society 2012, 56, 207-211, https://doi.org/10.29356/jmcs.v56i2.323. 
45. Monajjemi, M.; Bagheri, S.; Moosavi, M.S. Symmetry breaking of B2N(-,0,+): An aspect of the electric potential and atomic charges. Molecules 2015, 20, 21636-21657, https://doi.org/10.3390/molecules201219769.

46. Monajjemi, M.; Mohammadian, N.T. S-NICS: An aromaticity criterion for nano molecules. Journal of Computational and Theoretical Nanoscience 2015, 12, 4895-4914, https://doi.org/10.1166/jctn.2015.4458.

47. Monajjemi, M.; Ketabi, S.; Hashemian, Z.M.; Amiri, A. Simulation of DNA bases in water: Comparison of the Monte Carlo algorithm with molecular mechanics force fields. Biochemistry (Moscow) 2006, 71, 1-8, https://doi.org/10.1134/s0006297906130013.

48. Monajjemi, M.; Lee, V.S.; Khaleghian, M.; Honarparvar, B.; Mollaamin, F. Theoretical Description of Electromagnetic Nonbonded Interactions of Radical, Cationic, and Anionic NH2BHNBHNH2 Inside of the B18N18 Nanoring. J. Phys. Chem C 2010, 114, 15315-15330, https://doi.org/10.1021/jp104274z.

49. Monajjemi, M.; Boggs, J.E. A New Generation of BnNn Rings as a Supplement to Boron Nitride Tubes and Cages. J. Phys. Chem. A 2013, 117, 1670-1684, https://dx.doi.org/10.1021/jp312073q.

50. Monajjemi, M. Non bonded interaction between BnNn (stator) and BN B (rotor) systems: A quantum rotation in IR region. Chemical Physics 2013, 425, 29-45, https://doi.org/10.1016/j.chemphys.2013.07.014.

51. Monajjemi, M.; Robert, W.J.; Boggs, J.E. NMR contour maps as a new parameter of carboxyl's OH groups in amino acids recognition: A reason of tRNA-amino acid conjugation. Chemical Physics 2014, 433, 1-11, https://doi.org/10.1016/j.chemphys.2014.01.017.

52. Monajjemi, M. Quantum investigation of non-bonded interaction between the B15N15 ring and BH2NBH2 (radical, cation, and anion) systems: a nano molecularmotor. Struct Chem 2012, 23, 551-580, https://dx.doi.org/10.1007/s11224-011-9895-8.

53. Monajjemi, M. Metal-doped graphene layers composed with boron nitride-graphene as an insulator: a nanocapacitor. Journal of Molecular Modeling 2014, 20, 2507, https://link.springer.com/article/10.1007/s00894-014-2507-y.

54. Monajjemi, M.; Heshmat, M.; Haeri, H.H.; Kaveh, F. Theoretical study of vitamin properties from combined QM-MM methods: Comparison of chemical shifts and energy. Russian Journal of Physical Chemistry 2006, 80, 1061-1068, https://doi.org/10.1134/S0036024406070119.

55. Monajjemi, M.; Honaparvar, B.; Khalili Hadad, B.; Ilkhani, A.; Mollaamin, F. Thermo-Chemical Investigation and NBO Analysis of Some anxileotic as Nano- Drugs. African journal of pharmacy and pharmacology 2010, 4, 521-529, https://doi.org/10.33263/BRIAC106.66956705.

56. Monajjemi, M.; Najafpour, J.; Mollaamin, F. (3,3)4 Armchair carbon nanotube in connection with PNP and NPN junctions: Ab Initio and DFT-based studies. Fullerenes Nanotubes and Carbon Nanostructures 2013, 21, 213-232, https://doi.org/10.1080/1536383X.2011.597010.

57. Monajjemi, M., Jafari Azan, M., Mollaamin, F. Density functional theory study on B30N20 nanocage in structural properties and thermochemical outlook. Fullerenes Nanotubes and Carbon Nanostructures 2013, 21(6), 503-515, https://doi.org/10.1080/1536383X.2011.629762.

58. Monajjemi, M.; Ghiasi, R.; Ketabi, S.; Passdar, H.; Mollaamin, F. A Theoretical Study of Metal-Stabilised Rare Tautomers Stability: N4 Metalated Cytosine $\left(\mathrm{M}=\mathrm{Be}^{2+}, \mathrm{Mg}^{2+}, \mathrm{Ca}^{2+}, \mathrm{Sr}^{2+}\right.$ and $\left.\mathrm{Ba}^{2+}\right)$ in Gas Phase and Different. Journal of Chemical Research 2004, 1, 11-18, https://doi.org/10.3184/030823404323000648.

59. Monajjemi, M.; Baheri, H.; Mollaamin, F. A percolation model for carbon nanotube-polymer composites using the Mandelbrot-Given. Journal of Structural Chemistry 2011, 52, 54-59, https://doi.org/10.1134/S0022476611010070.

60. Dong, E.; Du, H.; Gardner, L. An Interactive Web-Based Dashboard to Track COVID-19 in Real Time. Lancet Infect. Dis. 2020, 20, 533-534, https://doi.org/10.1016/S1473-3099(20)30120-1.

61. Neto, M.L.R.; Almeida, H.G.; Esmeraldo, J.D.; Nobre, C.B.; Pinheiro, W.R.; de Oliveira, C.R.T.; da Costa Sousa, I.; Lima, O.M.M.L.; Lima, N.N.R.; Moreira, M.M.; et al. When Health Professionals Look Death in the Eye: The Mental Health of Professionals Who Deal Daily with the 2019 Coro-navirus Outbreak. Psychiatry Res. 2020, 288, 112972, https://doi.org/10.1016/j.psychres.2020.112972.

62. Adams, J.G.; Walls, R.M. Supporting the Health Care Workforce During the COVID-19 Global Epidemic. JAMA 2020, 323, 1439-1440, https://doi.org/10.1001/jama.2020.3972.

63. Dondorp, A.M.; Hayat, M.; Aryal, D.; Beane, A.; Schultz, M.J. Respiratory Support in COVID-19 Patients, with a Focus on Resource-Limited Settings. Am. J. Trop. Med. Hyg. 2020, 102, 1191-1197, https://doi.org/10.4269/ajtmh.20-0283.

64. Armocida, B.; Formenti, B.; Ussai, S.; Palestra, F.; Missoni, E. The Italian Health System and the COVID19 Challenge. Lancet Public Health 2020, 5, e253, https://doi.org/10.1016/S2468-2667(20)30074-8. 
65. . Szcze'sniak, D.; Gładka, A.; Misiak, B.; Cyran, A.; Rymaszewska, J. The SARS-CoV-2 and Mental Health: From Biological Mechanisms to Social Consequences. Prog. Neuro-Psychopharmacol. Biol. Psychiatry 2021, 104, 110046, https://doi.org/10.1016/j.pnpbp.2020.110046.

66. Ahmad, T.; Haroon; Baig, M.; Hui, J. Coronavirus Disease 2019 (COVID-19) Pandemic and Economic Impact. Pak. J. Med. Sci. 2020, 36, S73-S78, https://doi.org/10.12669/pjms.36.COVID19-S4.2638.

67. Autrán-Gómez, A.M.; Favorito, L.A. The Social, Economic and Sanitary Impact of COVID-19 Pandemic. Int. Braz J. Urol 2020, 46, 3-5, https://doi.org/10.1590/S1677-5538.IBJU.2020.S1ED2.

68. Machado, B.A.S.; Hodel, K.V.S.; Barbosa-Júnior, V.G.; Soares, M.B.P.; Badaró, R. The Main Molecular and Serological Methods for Diagnosing COVID-19: An Overview Based on the Literature. Viruses 2020, 13, 40, https://doi.org/10.3390/v13010040.

69. Cheng, M.P.; Papenburg, J.; Desjardins, M.; Kanjilal, S.; Quach, C.; Libman, M.; Dittrich, S.; Yansouni, C.P. Diagnostic Testing for Severe Acute Respiratory Syndrome-Related Coronavirus 2. Ann. Intern. Med. 2020, 172, 726-734, https://doi.org/10.7326/M20-1301.

70. Lu, N.; Cheng, K.W.; Qamar, N.; Huang, K.C.; Johnson, J.A. Weathering COVID-19 Storm: Successful Control Measures of Five Asian Countries. Am. J. Infect. Control. 2020, 48, 851-852, https://doi.org/10.1016/j.ajic.2020.04.021.

71. 12. Cha, V. Asia's COVID-19 Lessons for the West: Public Goods, Privacy, and Social Tagging. Wash. Q. 2020, 43, 33-50, https://doi.org/10.1080/0163660X.2020.1770959.

72. Rai, P.; Kumar, B.K.; Deekshit, V.K.; Karunasagar, I.; Karunasagar, I. Detection Technologies and Recent Developments in the Diagnosis of COVID-19 Infection. Appl. Microbiol. Biotechnol. 2021, 441-455, https://doi.org/10.1007/s00253-020-11061-5.

73. Loeffelholz, M.J.; Tang, Y.W. Laboratory Diagnosis of Emerging Human Coronavirus Infections-the State of the Art. Emerg. Microbes Infect. 2020, 9, 747-756, https://doi.org/10.1080/22221751.2020.1745095.

74. Lippi, G.; Simundic, A.M.; Plebani, M. Potential Preanalytical and Analytical Vulnerabilities in the Laboratory Diagnosis of Coronavirus Disease 2019 (COVID-19). Clin. Chem. Lab. Med. 2020, 58, 10701076, https://doi.org/0.1515/cclm-2020-0285.

75. Afzal, A. Molecular Diagnostic Technologies for COVID-19: Limitations and Challenges. J. Adv. Res. 2020, 26, 149-159. Genes 2021, 12, 565, https://doi.org/10.1016/j.jare.2020.08.002.

76. Shen, L.; Zhang, Z.; He, F. The Phylogenetic Relationship Within SARS-CoV-2s: An Expanding Basal Clade. Mol. Phylogenet. Evol. 2020, 107017, https://doi.org/10.1016/j.ympev.2020.107017.

77. Giovanetti, M.; Benedetti, F.; Campisi, G.; Ciccozzi, A.; Fabris, S.; Ceccarelli, G.; Tambone, V.; Caruso, A.; Angeletti, S.; Zella, D.; et al. Evolution Patterns of SARS-CoV-2: Snapshot on its Genome Variants. Biochem. Biophys. Res. Commun. 2020, 538, 88-91, 10, https://doi.org/10.1016/j.bbrc.2020.10.102.

78. Abdel-Moneim, A.S.; Abdelwhab, E.M.; Memish, Z.A. Insights into SARS-CoV-2 Evolution, Potential Antivirals, and Vaccines. Virology 2021, 558, 1-12, https://doi.org/10.1016/j.virol.2021.02.007.

79. Gouv, W. Biomedicine \& Pharmacotherapy Impact of Virus Genetic Variability and Host Immunity for the Success of COVID-19 Vaccines. Biomed Pharm. 2021, 136, https://doi.org/10.1016/j.biopha.2021.111272.

80. Van Poelvoorde, L.A.E.; Saelens, X.; Thomas, I.; Roosens, N.H. Next-Generation Sequencing: An EyeOpener for the Surveillance of Antiviral Resistance in Influenza. Trends Biotechnol. 2020, 38, 360-367, https://doi.org/10.1016/j.tibtech.2019.09.009.

81. Kemp, S.A.; Collier, D.A.; Datir, R.P.; Ferreira, I.A.T.M.; Gayed, S.; Jahun, A.; Hosmillo, M.; Rees-Spear, C.; Mlcochova, P.; Lumb, I.U.; et al. SARS-CoV-2 Evolution During Treatment of Chronic Infection. Nature 2021, 592, 277-282, https://www.nature.com/articles/s41586-021-03291-y.

82. Bazykin, G.; Stanevich, O.; Danilenko, D.; Fadeev, A.; Komissarova, K.; Ivanova, A.; Sergeeva, M.; Safina, K.; Nabieva, E.; Klink, G.; et al. Emergence of Y453F and $\Delta 69-70 H V$ Mutations in a Lymphoma Patient with Long-Term COVID-19. https://virological.org/t/emergence-of-y453f-and-69-70hv-mutations-in-alymphoma-patient-with-long-term-covid-19/580. 